\title{
Como estimar a importância relativa de um único fator em estudos de aceitação via Conjoint Analysis?
}

Bruna Borges SILVA ${ }^{1}$

Eric Batista FERREIRA ${ }^{2}$

Adriana DIAS 3

${ }^{1}$ Graduanda em Matemática Licenciatura pela Universidade Federal de Alfenas (UNIFAL-MG), bruna_borges_20@hotmail.com

${ }^{2}$ Doutorado em Estatística e Experimentação Agropecuária pela Universidade Federal de Lavras (UFLA, 2007), Pósdoutorado em Estatística Multivariada (UFLA, 2009) e Pós-doutorado em Sensometria (NOFIMA, 2013), Professor Adjunto IV do Instituto de Ciências Exatas (ICEx) da Universidade Federal de Alfenas (UNIFAL-MG)

${ }^{3}$ Doutorado em Estatística e Experimentação Agropecuária pela Universidade Federal de Lavras (UFLA,2014), Professor Adjunto I do Instituto de Ciências Exatas (ICEx) da Universidade Federal de Alfenas (UNIFAL-MG)

Recebido em: 07/05/2015 - Aprovado em: 30/10/2015 - Disponibilizado em: 31/10/2015

Resumo: No meio comercial é constante a concorrência de empresas e de gerentes de marketing para atraírem clientes. As indústrias procuram cada vez mais aprimorarem seus produtos e oferecerem o que têm de melhor, sempre visando o sucesso e a lucratividade. Dentro desse contexto a técnica estatística chamada Conjoint Analysis (Análise Conjunta) pode prever a aceitação do consumidor em relação a determinado produto ou serviço estimando os Coeficientes de Preferência de cada nível dos fatores (atributos), fornecendo assim a Importância Relativa dos fatores (características) desse produto. Porém em estudos em que um único fator é avaliado, não é possível obter essa estimativa para este fator. Neste trabalho objetivou-se propor uma forma de estimar a Importância Relativa de um único fator em estudos de aceitação e ilustrar o método com um estudo de marcas comerciais de hambúrgueres vendidas no município de Alfenas. De acordo com os resultados obtidos, o fator marca foi responsável por 17,87\% da aceitação.

Palavras-chave: Preferência. Análise sensorial. Hambúrguer. Consumidores. Regressão linear múltipla.

Abstract: In the competitive market commercial managers fight for attracting customers. The industries are increasingly looking to turn their products better, always seeking success and profitability. In this context the Conjoint Analysis can predict consumer acceptation in relation of a given product or service, estimating the preference coefficients of each level of the factors (attributes), therefore providing relative importance for each factor (characteristic) of the product. However, in studies where a single factor is evaluated, it is not common to estimate its relative importance (since it is relative). This work aimed to propose a way to estimate the relative importance of a single factor in acceptance of study and illustrate the method with a study of commercial brands of hamburger sold in Alfenas, Brazil. According to the results obtained, the factor brand was responsible for $17.87 \%$ of the acceptance.

Keywords: Preference. Sensory analysis. Hamburger. Consumers. Multiple linear regression.

\section{INTRODUÇÃ̃}

Na indústria de alimentos, além dos fatores sensoriais, os consumidores analisam fatores como: embalagem, preço, rótulo, marca, ou características relacionadas à sua preferência; os fatores não sensoriais. A influência das características externas do alimento é muito significativa e mostra que para obter êxito na fabricação do alimento, tanto as características sensoriais quanto as não-sensoriais devem ser analisadas. (DELLA LUCIA, 2008).

$$
\text { A embalagem dos produtos, }
$$
principalmente de alimentos, tem sido mais avaliada nos últimos anos. Os consumidores a consideram um fator muito importante na hora da compra, pois as principais informações sobre o produto estão na embalagem. Segundo Carneiro (2002), alguns atributos da 
embalagem que afetam a decisão de compra do consumidor são, por exemplo, a data de validade, informação nutricional, lista de ingredientes, peso líquido e modo de preparo.

A Conjoint Analysis é uma metodologia estatística usada para analisar a preferência do consumidor em relação a algum produto ou serviço oferecido. Cada produto possui um ou vários fatores (características) relacionados a ele, e é por meio desses fatores que os consumidores decidem sua preferência. Pretto e Artes (2009) explicam que cada fator pode ser dividido em diferentes níveis, e a combinação dos níveis com seus respectivos fatores geram os chamados estímulos (tratamentos).

Muitos estudos já foram realizados com o uso da técnica Conjoint Analysis, desde seu surgimento na década de 70. Acredita-se que esse deve ser o desenvolvimento metodológico mais importante em pesquisa de marketing nos últimos quarenta e cinco anos. A Conjoint Analysis se destaca pela capacidade de prever as reações dos compradores e, para isso, são usados simuladores de mercado. Além disso, essa técnica contribui para otimizar os produtos/serviços e aumentar o lucro das empresas (RAO; PILLI, 2014).

Segundo Bastos (2010), os gerentes de marketing enfrentam dificuldades na previsão de vendas e criação de novos produtos, ou até mesmo para aprimorarem produtos já existentes. É nesse contexto que a técnica Conjoint Analysis pode auxiliar as empresas e comerciantes, prevendo questões como: lucratividade, impacto de novos produtos e necessidade de mudança.

Carneiro et al. (2012) utilizaram a técnica Conjoint Analysis para analisar os fatores da embalagem e do rótulo de cachaça que influenciam na decisão de compra do consumidor. Na simulação do mercado de cachaça, feita por meio dessa técnica, foram avaliados dois níveis dos seguintes fatores: tipo de madeira, tempo de envelhecimento, marca, ilustração do rótulo e tipo de embalagem, e concluiu-se que os mesmos influenciam a decisão dos consumidores de cachaça. A embalagem preferida foi a garrafa de vidro transparente com capacidade de 700 $\mathrm{mL}$, com tampa metálica rosqueável, com rótulo contendo ilustração não relacionada ao processo de produção de cachaça e informação "envelhecida por 36 meses em tonéis de carvalho".

Dantas et al. (2005) avaliaram o impacto das características da embalagem na intenção de compra de couve minimamente processada. Foram avaliados cinco fatores da embalagem da couve: informação, tipo de produção, cor, preço e visibilidade do produto. Dentre eles, o fator que mais influenciou na decisão dos consumidores foi a informação.

Della Lucia et al. (2007) utilizaram a Conjoint Analysis para avaliar o efeito de alguns fatores da embalagem de café orgânico na intenção de compra dos consumidores. Dos quatro fatores avaliados: preço, cor da embalagem, marca e informação geral sobre 
orgânicos, o que mais influenciou na intenção de compra foi a marca.

A Conjoint Analysis tradicional estima a Importância Relativa de dois ou mais fatores que compõem um produto ou serviço. Sendo assim, em estudos com um único fator se torna necessária uma alternativa para estimar a importância do mesmo. Este trabalho teve como objetivo propor uma forma de estimar a Importância Relativa (IR) de um único fator em estudos de aceitação e ilustrar o método com um experimento de marcas comerciais de hambúrguer vendidas no município de Alfenas $-\mathrm{MG}$.

\section{METODOLOGIA}

Os dados utilizados neste trabalho são provenientes de um estudo sobre a caracterização e aceitação de hambúrgueres, realizado em 2013 pela Professora Flávia Della Lucia, na Faculdade de Nutrição da Universidade Federal de Alfenas.

A avaliação sensorial foi realizada por 122 consumidores que provaram hambúrgueres bovinos e de frango de 5 marcas comerciais (chamadas aqui de A, B, C, D e E).

Os consumidores preencheram uma ficha individual de avaliação para cada amostra. A escala hedônica de 9 pontos foi utilizada para as avaliações, sendo os extremos da escala: o valor 1, correspondente ao termo hedônico "desgostei extremamente", e o valor 9, correspondente ao termo hedônico "gostei extremamente."

O experimento foi conduzido em delineamento em blocos casualizados (DBC) completos, segundo um modelo misto, em que a fonte de variação marca foi considerada um fator fixo e os consumidores (blocos), um fator aleatório:

$$
y_{i j}=\mu+\tau_{i}+\beta_{j}+e_{i j}
$$

em que $y_{i j}$ é a aceitação do produto $i$ pela pessoa $j ; \mu$ é uma constante comum a todas as observações; $\tau_{i}$ é o efeito fixo da $i$-ésima marca; $\beta_{j}$ é o efeito aleatório do j-ésimo consumidor, assumindo-se que $\beta \sim \mathrm{N}\left(0, \sigma_{b}^{2}\right)$; $\varepsilon_{i j}$ é o erro aleatório associado a $y_{i j}$, assumindose que $\beta \sim \mathrm{N}\left(0, \sigma_{e}^{2}\right)$.

\subsection{Conjoint Analysis baseada em notas}

De acordo com Resende e Nakano (2015), a Conjoint Analysis baseada em notas utiliza o modelo de regressão linear múltipla. Os tratamentos são formados pela combinação dos níveis de cada fator. Assim, no caso de $r$ fatores, com $m_{s}$ níveis tem-se um total de $J=\prod_{s=1}^{r} m_{s}$ tratamentos.

O modelo de utilidade para a Conjoint Analysis é:

$$
U_{n j}=\beta_{0}+\sum_{s=1}^{r} \sum_{i=1}^{m_{s}} X_{s i}^{j} \beta_{s i}+\varepsilon_{n j}
$$

em que: 
- $U_{n j}$ é a nota de preferência, aceitação ou intenção de compra dado o $j$-ésimo tratamento pelo $n$-ésimo consumidor;

- $\beta_{0}$ é o intercepto (nota média de todos os tratamentos);

- $\beta_{s i}$ é o Coeficiente de Preferência associado ao $i$-ésimo nível do $s$-ésimo fator;

- $\varepsilon_{n j}$ é o erro aleatório não observável do modelo.

$X_{s i}^{j}=1$ quando o o $i$-ésimo nível do $s$-ésimo fator está presente no $j$-ésimo tratamento e $X_{s i}^{j}=0$ caso contrário.

O método dos mínimos quadrados ordinários pode ser utilizado para estimar os parâmetros do modelo. Em algumas situações, para completar o posto da matriz $X$, utiliza-se a restrição $\sum_{i=1}^{m i} \beta_{s i}=0$. Dessa forma, o sistema de equações normais, $X^{\prime} X \hat{\beta}=X^{\prime} Y$, apresenta solução única e é possível interpretar as estimativas dos parâmetros da seguinte forma:

- $\beta_{s i}<0$ representa efeito desfavorável do nível do fator, ou seja, diminui a nota de preferência pelo tratamento;

- $\beta_{s i}>0$ representa efeito favorável na preferência do consumidor.

Quando essa restrição não é imposta, a solução do sistema é obtida pela utilização de uma inversa generalizada da matriz $X$.
Neste trabalho avaliou-se um único fator, marca de hambúrgueres, com 5 níveis, A, B, C, D e E.

\subsection{Regressão por variáveis dummy}

Charnet et al. (2008) explicam que independentemente do modelo de regressão utilizado (simples ou múltiplo), pode-se lidar com variáveis qualitativas (nominais). Para inserir alguma variável qualitativa no modelo é necessário criar uma ou mais variáveis numéricas, denominadas variáveis fictícias (variáveis “dummy”).

Segundo Missio e Jacobi (2007), podese criar variáveis fictícias para quantificar os atributos que assumirão os valores 1 ou 0 , indicando, respectivamente, a presença ou ausência destes. Os modelos de regressão que possuem apenas variáveis qualitativas são chamados de modelos de análise de variância (ANOVA).

\subsection{Importância Relativa}

Uma vantagem da Conjoint Analysis é a estimação da Importância Relativa (IR) de cada fator que compõe os tratamentos analisados. A partir dessas estimativas pode-se decidir quais fatores devem ser mais focados na avaliação.

O impacto ou o efeito que o fator tem sobre a aceitação do produto pelo consumidor pode ser medido pela estimação da importância relativa de cada fator. 
Barbosa e Silva (2014) explicam que os valores $\beta_{\text {si }}$ para $i=1,2, \ldots, m_{s}$ fornecem a estimativa da Importância do $s$-ésimo fator dada por: $\hat{I}_{s}=\max \left(\hat{\beta}_{s i}\right)-\min \left(\hat{\beta}_{s i}\right)$.

A estimativa da Importância Relativa (IR) de cada fator será:

$$
\widehat{I R}_{S}(\%)=\frac{\hat{I}_{s}}{\sum_{S=1}^{r} \hat{I}_{S}} \cdot 100
$$

Em situações em que apenas um fator é avaliado, não é possível calcular a Importância Relativa pelo uso da fórmula apresentada anteriormente. Como alternativa, propõe-se que o cálculo da Importância Relativa para essas situações, seja realizado considerando a seguinte fórmula:

$$
I_{f}(\%)=\frac{I_{f}}{A} \cdot 100
$$

em que $I R_{f}$ é a importância relativa do fator avaliado; $A$ representa a amplitude da escala hedônica utilizada para avaliação dos tratamentos.

\section{RESULTADOS E DISCUSSÃO}

A análise estatística dos dados foi realizada no software estatístico R (R CORE TEAM, 2015).

O método dos mínimos quadrados ordinários foi utilizado para estimar os parâmetros do modelo de regressão. Como o fator em estudo, marca, é uma variável categórica e apresenta 5 níveis (A, B, C, D e
E), foram utilizadas 5 variáveis "dummy" para representar cada nível do fator.

Para obter a solução do sistema de equações normais, foi necessário utilizar uma inversa generalizada para a matriz $\mathrm{X}$, obtida por meio da utilização da função ginv.

O modelo ajustado foi o seguinte:

$$
\begin{gathered}
\hat{Y}=5,56+0,35 X_{1}+1,15 X_{2}+1,77 X_{3} \\
+1,25 X_{4}+1,04 X_{5}
\end{gathered}
$$

Em que,

- $X_{1}$ representa a marca A;

- $X_{2}$ representa a marca B;

- $X_{3}$ representa a marca C;

- $X_{4}$ representa a marca $\mathrm{D}$;

- $X_{5}$ representa a marca E;

As estimativas associadas a cada variável representam o efeito da marca na nota de aceitação dada pelos consumidores aos tratamentos avaliados. A marca $\mathrm{C}$ apresentou maior nota de aceitação $(5,56+1,77=7,33)$ enquanto a marca $A$, apresentou a menor $(5,56$ $+0,35=5,90)$.

A análise de variância do experimento foi realizada utilizando-se o pacote ExpDes.pt (FERREIRA; CAVALCANTI; NOGUEIRA, 2014) e o teste Tukey foi utilizado para comparar as notas médias de aceitação das marcas (Tabela 1). 
Tabela 1 - Comparação entre as notas médias das 5 marcas comerciais de hambúrgueres

\begin{tabular}{|ccc|}
\hline Marcas & Médias* & \\
\hline C & $\mathbf{7 , 3 3}$ & a \\
\hline D & $\mathbf{6 , 8 1}$ & ab \\
\hline B & $\mathbf{6 , 7 1}$ & b \\
\hline E & $\mathbf{6 , 6 0}$ & b \\
\hline A & $\mathbf{5 , 9 0}$ & c \\
\hline
\end{tabular}

*Médias de tratamentos seguidas por pelo menos uma mesma letra não diferem estatisticamente entre si $(p>0,05)$ pelo teste Tukey.

Ao se utilizar o método proposto para estimar a Importância Relativa do fator em estudo, considerando: $A=8$ ( $9-1$ pontos da escala hedônica), $\hat{\beta}_{\max }=1,77$ e $\hat{\beta}_{\text {min }}=0,35$, obteve-se a importância relativa de 17,87\% para o fator marca.

No estudo de Della Lucia et al. (2007) sobre o efeito dos fatores da embalagem de café orgânico torrado e moído, na intenção de compras dos consumidores, a marca apresentou importância relativa superior a $11 \%$.

Carneiro et al. (2002) estudaram o impacto da embalagem de óleo de soja na intenção de compra do consumidor e constataram que para $50 \%$ dos consumidores de dois dos quatro grupos avaliadores envolvidos nesse estudo, a marca do produto apresentou Importância Relativa superior a $10 \%$.

O método proposto é útil para estimar a Importância Relativa quando um único fator é avaliado em estudos de aceitação de consumidores, o que não seria possível ao se utilizar o estimador da Importância Relativa tradicionalmente utilizado em estudos com a Conjoint Analysis.

\section{CONCLUSÃO}

O método pode ser utilizado para estimar a Importância Relativa em estudos de aceitação em que é avaliado um único fator.

Com a utilização do método proposto foi possível estimar que o fator marca é responsável por $17,87 \%$ da aceitação de hambúrgueres vendidos no município de Alfenas, MG.

\section{REFERÊNCIAS}

BARBOSA, E. C.; SILVA, C. H. O. Avaliação de uma disciplina básica de Estatística por Conjoint Analysis. Revista de Estatística UFOP, v.3, p. 270-274, 2014.

\section{BASTOS, F. S. Análise Conjunta de Fatores}

\section{Baseada em Escolhas: Estimação e}

Inferências. 2010. 82 f. Dissertação (Mestrado em Estatística Aplicada e Biometria),

Departamento de Estatística, Universidade Federal de Viçosa, Viçosa , 2010.

\section{CARNEIRO, J. D. S. Impacto da}

Embalagem de Óleo de Soja na Intenção de Compra do Consumidor via Conjoint Analysis. 2002. 74 f. Dissertação (Mestrado em Ciência e Tecnologia de Alimentos) - 
Departamento de Tecnologia e Alimentos,

Universidade Federal de Viçosa, Viçosa, 2002.

CARNEIRO, J. D. S. et al. Sugarcane spirit market share simulation: an application of conjoint analysis. Ciência e Tecnologia de Alimentos, Campinas, v.32, n.4, p.645-652, out./dez. 2012.

CHARNET, R. et al. Análise de Modelos de

Regressão Linear: com aplicações. 2.ed.

Campinas: UNICAMP, 2008. 356 p.

DANTAS, M. I. S. et al. Avaliação na intenção de compra de couve minimamente processada. Ciência e Tecnologia de Alimentos, Campinas, v.35, n.4, p.762-767, out./dez. 2005.

DELLA LUCIA, S. M. et al. Fatores da embalagem de café orgânico torrado e moído na intenção de compra do consumidor.

Ciência e Tecnologia de Alimentos,

Campinas, v.27, n.3, p.485-491, jul./set. 2007.

\section{DELLA LUCIA, S. M. Métodos Estatísticos} para a Avaliação da Influência de

\section{Características não Sensoriais no}

Comportamento do Consumidor. 2008. 135

f. Tese (Doutorado em Ciência e Tecnologia de Alimentos) - Departamento de Tecnologia e Alimentos, Universidade Federal de Viçosa, Viçosa, 2008.
FERREIRA, E. B.; CAVALCANTI, P. P.; NOGUEIRA, D. A. ExpDes: An R package for ANOVA and Experimental Designs.

Applied Mathematics, v. 5, p. 2952-2958. 2014.

MISSIO, F.; JACOBI, L.F. Variáveis dummy: especificações de modelos com parâmetros variáveis. Ciência e Natura, v. 29, n. 1, p.111 - 135, 2007.

PRETTO, K.; ARTES, R. Análise de Preferência Conjunta: Um Estudo Sobre a Omissão de Atributo. Revista Brasileira de Estatística, Rio de janeiro, v. 70, n.233, p. 731, jul./dez. 2009.

\section{R CORE TEAM. R: A language and} environment for statistical computing. $R$ Foundation for Statistical Computing, Vienna, Austria. 2015.

RAO, V. R.; PILLI, L. E. Conjoint Analysis para pesquisa de marketing no Brasil. Revista Brasileira de Marketing, v.13, n.4, p. 25-38, set. 2014.

RESENDE, V. S.; NAKANO, E. Y. Análise conjunta baseada em notas via modelo de regressão beta. Revista Brasileira Biometria, São Paulo, v.33, n.1, p.51-62, 20 